\title{
3D Instantaneous Reconstruction of Turbulent Industrial Flames Using Computed Tomography of Chemiluminescence (CTC)
}

\author{
A. Unterberger $\left({ }^{1},{ }_{1}^{1}\right.$ M. Röder, ${ }^{2}$ A. Giese, ${ }^{2}$ A. Al-Halbouni, ${ }^{2}$ A. Kempf $\left({ }^{1},{ }^{1}\right.$ and K. Mohri ${ }^{1}$ \\ ${ }^{1}$ Institute for Combustion and Gas Dynamics (IVG) - Chair of Fluid Dynamics, University of Duisburg-Essen, Germany \\ ${ }^{2}$ Gas- und Wärme-Institut Essen e. V. (GWI), Essen, Germany \\ Correspondence should be addressed to A. Unterberger; andreas.unterberger@uni-due.de
}

Received 6 July 2018; Accepted 16 September 2018; Published 29 October 2018

Guest Editor: Xiaoke Ku

Copyright (C) 2018 A. Unterberger et al. This is an open access article distributed under the Creative Commons Attribution License, which permits unrestricted use, distribution, and reproduction in any medium, provided the original work is properly cited.

Computed Tomography of Chemiluminescence (CTC) was used to reconstruct the instantaneous three-dimensional (3D) chemiluminescence field of a high-power industrial flame, which was made optically accessible, for the first time. The reconstruction used 24 projections that were measured simultaneously, in one plane and equiangularly spaced within a total fan angle of $172.5^{\circ}$. The 3D results were examined by plotting both vertical and horizontal slices, revealing highly wrinkled structures with good clarity. The results presented are one of a series of experimental demonstrations of CTC applications to turbulent gaseous flames. The work reveals the potential to use any kind of luminescence measurement, such as emission from heated particles in coal-fired flames, for analysis of the flame shape directly in 3D.

\section{Introduction}

The use of fossil fuels such as coal remains to be the main source in today's power generation and likely to continue being so for the coming decades. However, conventional coal combustion is accompanied with harmful pollutants that disturb our environment and contribute to global warming. Hence, much scientific effort and investment is being increasingly dedicated to the development of cleaner and more efficient coal-burning technologies. In this context, there is a need for advanced monitoring techniques for the relevant flames, which are typically turbulent and inherently unsteady and three-dimensional (3D). Therefore, instantaneous volumetric data is key to obtaining in-depth knowledge of such flames to facilitate optimising coal combustion efficiency with respect to energy and pollutant production. The flame shape and expansion as well as temporal fluctuations, e.g., combustion instabilities, are examples of important information that will aid in the development and optimisation of the thermal processes involved.
Several non-intrusive flame diagnostic techniques such as laser-based ones currently exist, which were originally developed to deliver planar information. In principle, it is possible to use complex experiments constituting highspeed cameras, lasers, and rotating mirrors, to obtain timeresolved 3D information about species and temperature from multiple quasi-instantaneous light-sheet measurements $[1,2]$. Nonetheless, this approach is very expensive and challenging. On the other hand, the CTC technique $[3,4]$ can calculate at least the instantaneous spatially and temporally resolved flame shape information directly in $3 \mathrm{D}$, using comparatively simpler and less costly arrangements. The CTC utilises flame chemiluminescence measurements, and hence there is no need for an external source of illumination. Since chemiluminescence occurs in a region close to the reaction zone, the reconstructed 3D chemiluminescence field can reveal important geometrical features such as flame propagation and wrinkling, flame orientation, vortex shedding and breakdown, jet precession and recirculation and local quenching. In principle, any kind of emission measurement, 


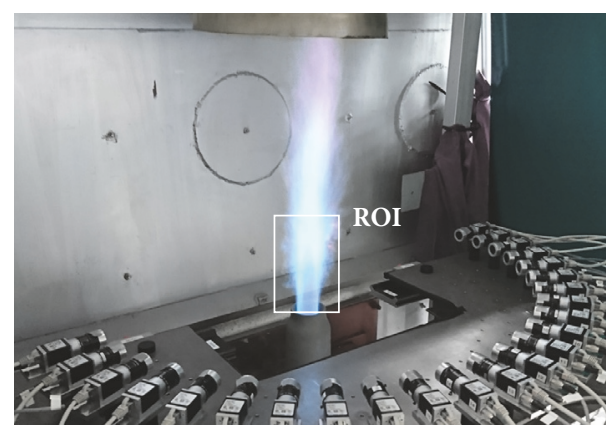

FIgURE 1: The setup used for the CTC, constituting 24 cameras with filters (Schott BG40), arranged equiangularly within a $172.5^{\circ}$ region, around the burner operated with natural gas, at the GWI (the white box shows the imaging region of interest ROI).

such as emissions from heated soot particles in the flame, as demonstrated by Hossain et al. [5] can be used with the CTC. Our focus on gaseous flames so far demonstrates the capability of CTC and prepares the technique for application to coal-fired combustion where emissions from heated coal particles will be used for volumetric flame reconstructions [6]. The CTC was first developed to reconstruct the instantaneous chemiluminescence field of gaseous flames by Floyd [7], and was proven to work using commodity cameras. It was initially tested using several phantoms (exactly known fields which are compared to their reconstruction for quantified analysis of the reconstruction quality). Some flame experiments, where either one camera was rotated around a steady flame [8] or mirrors captured 10 flame chemiluminescence images for the reconstructions [3], were also demonstrated.

We have built a new setup that constitutes 24 low-cost $(<$ $€ 600)$ and light-weight ( $<90 \mathrm{~g}$ ) monochrome CCD cameras, for the application of CTC to a series of different flames. It was first applied to quasi-steady [9] and unsteady Bunsen flames [10], and a turbulent swirl flame [4] within our institute. To demonstrate the versatility of the technique at its current stage, we took the setup to the Gas- und Wärme-Institute (GWI) in Essen to reconstruct the chemiluminescence field of flames from a high-power industrial burner that was made optically accessible. Within three days, the setup was completed around the burner and flame reconstructions were achieved, revealing information about the flame shape, which are presented in this paper, for the first time.

\section{The CTC Technique}

The CTC directly calculates the instantaneous 3D chemiluminescence field using multiple measurements (in the form of camera images) that are obtained from different angles $\theta$ around the object, as depicted in Figure 1. The total number of pixels (or projections) $N_{p i x}$, in one camera image which is obtained at the same angle $\theta$, forms a view $\mathrm{q}$, with a total number of $N_{\text {cams }}$ views. The spectral density detected on the camera pixel corresponds to the sum (line-of-sight projection) of the light emitted along the light ray path
TABLE 1: The burner flow conditions: $\phi$ is the equivalence ratio, $\dot{V}_{\mathrm{CH}_{4}}$ and $\dot{V}_{\text {air }}$ are the methane and air volume flow rates of the combustion gases, respectively, $R e$ is the cold flow Reynolds number based on $D$, $t_{\text {exp }}$ is the camera exposure time and $P$ is the flame thermal power.

\begin{tabular}{lccccc}
\hline$\phi$ & $\dot{V}_{\mathrm{CH}_{4}}\left(\mathrm{~m}^{3} / \mathrm{h}\right)$ & $\dot{\mathrm{V}}_{\text {air }}\left(\mathrm{m}^{3} / \mathrm{h}\right)$ & $R e$ & $t_{\text {exp }}(\mathrm{ms})$ & $P(\mathrm{~kW})$ \\
\hline 0.79 & 8 & 100 & $\sim 40000$ & 0.1 & 83 \\
\hline 0.79 & 10 & 127 & $\sim 50000$ & 0.1 & 105 \\
\hline
\end{tabular}

through the probe volume. This is based on the fundamental radiative transfer equation (RTE), which relates the change in radiation intensity along a ray path to local absorption and volume emission [11, 12]. In the CTC, the RTE is simplified by neglecting scattering and re-absorption. The projection measurement $I_{q p}$ is approximated as a finite sum going through the $3 \mathrm{D}$ field, that is, discretised into a total number of $N_{v o x}$ voxels, via (1). In (1) $w_{q p v}$ represents the contribution of each voxel $v$ to the line of sight projection $\mathrm{p}$ of view $\mathrm{q}$ and $Q_{v}(x, y, z)$ is the scalar field to be reconstructed.

$$
I_{q p}=\sum_{v=1}^{N_{v o x}} w_{q p v} Q_{v}
$$

The CTC algorithm that uses the projection measurements is based on the iterative Algebraic Reconstruction Technique (ART) [13]. The camera optics are modelled via simple raytracing representation, and non-parallel rays were implemented to account for perspective effects. This means that the rays fan out as a function of focal length and cover a large volume focused in the focal plane as a function of focal depth, thus requiring a direct reconstruction in $3 \mathrm{D}$, as opposed to stacked reconstructions in $2 \mathrm{D}$. The projection rays also consider the blur-effect, to account for limited depth of field. For the reconstruction process, the measured projections $I_{q p}$ are compared to equivalent projections that are taken through the current iteration's estimate of the field $Q_{v}^{h}$, where $h$ denotes the current iteration step. The reconstruction is assumed to be converged once the absolute difference of the sum of the field vector, from one iteration to the next, is below the threshold $\Delta_{c} \times Q_{v}^{h}$. The value of $\Delta_{c}$ is chosen by the user and is typically in the range of $10^{-3}$ and $10^{-6}$. In-depth detail on the algorithm and initial extensive parametric phantom studies can be found in $[3,7,8]$.

\section{The Experimental Setup}

A standard industrial burner was used, which is commonly utilised for heating purposes in thermal processes. The burner is modular and can be equipped with different nozzle configurations. The ceramic nozzle head used here had an exit diameter of $D=65 \mathrm{~mm}$ and length $L=300 \mathrm{~mm}$. The burner was not encased and the premixed combustion gases constituted natural gas (90 mol\% methane) and air at atmospheric conditions. Premixing was achieved inside the ceramic nozzle. The chemiluminescences images were obtained for the flame operating with a thermal power of 83 $\mathrm{kW}$ and $105 \mathrm{~kW}$. The complete test conditions are given in Table 1. 


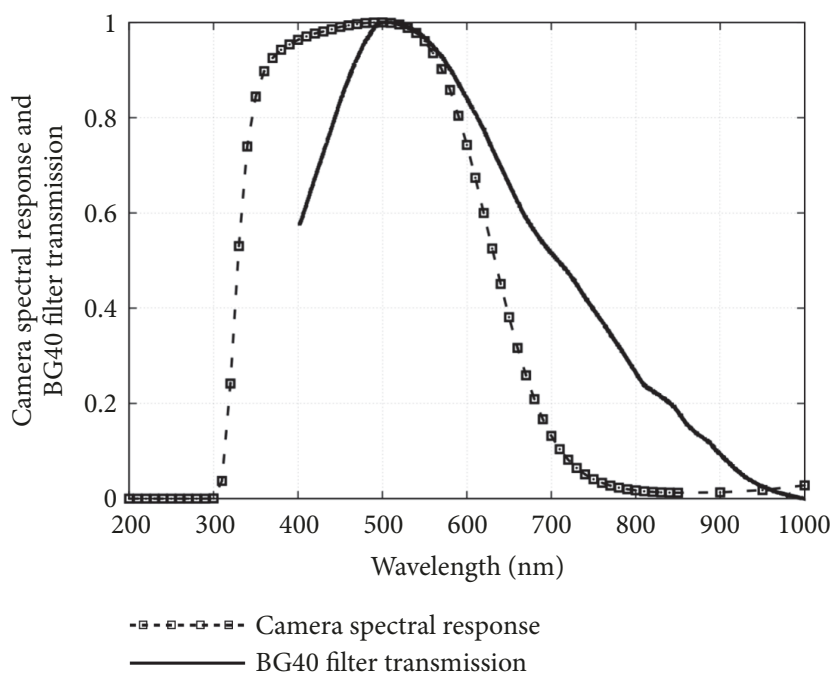

FIGURE 2: Spectral response of the cameras and the transmission curve of the Schott BG40 filter used for water emission suppression.

As shown in Figure 1, a total of $N_{\text {cams }}=24$ CCD cameras (Basler acA645-100gm containing a 0.5" Sony ICX414 monochrome sensor, 659 by 494 pixels of size $9.9 \times 9.9 \mu \mathrm{m})$ were mounted on one plate, with a constant angular separation of $\theta=7.5^{\circ}$ in one plane within a total fan angle of $172.5^{\circ}$ around the burner. Preset holes at a fixed distance from the burner and the aforementioned angular separation were used for the camera mounts. A back-illuminated pinhole, which was mounted on a rotation stage that measures the angle to within $\pm 0.5^{\circ}$ accuracy, was placed at the burner location. Camera alignment was achieved by first lining the point light onto camera 1 and consecutively rotating it by $7.5^{\circ}$ to point to the rest of the cameras. Each time the relevant camera was adjusted to ensure that the image of the light point falls on the centre pixel of the camera image. The adequacy of this camera alignment method was checked in our previous work [4].

The spectral response of the cameras, at $>60 \%$, is between about $400 \mathrm{~nm}$ and $680 \mathrm{~nm}$. Kowa C-mount lenses (focal length $12 \mathrm{~mm}$ ) were used. Each camera was fitted with an optical filter (Schott BG40) to eliminate the detection of thermally heated water which emits lights in the near infra-red and infra-red range. The images were taken by capturing all other emitted signals from the excited species, the prominent ones being $\mathrm{CH} *$ (around $430 \mathrm{~nm}$ ) an $\mathrm{C}_{2} *$ (around $515 \mathrm{~nm}$ ) and broadband $\mathrm{CO}_{2} *$. Figure 2 illustrates the spectral response of the cameras and the transmission curve of the filters used. One trigger signal was sent to all cameras and the image readout was done simultaneously through two Ethernet switches (Gigabit smart TL-SG2424P) that were connected to the control and evaluation computer. The tomography setup used here was the same as the one used recently to reconstruct a turbulent swirl flame [4].

The interest was in lowering the camera exposure time $t_{\text {exp }}$ as much as possible to minimise motion blur, so that finer flame structures could be resolved. The aperture opening was set to its largest opening size, $f / 1.4$, and an exposure time

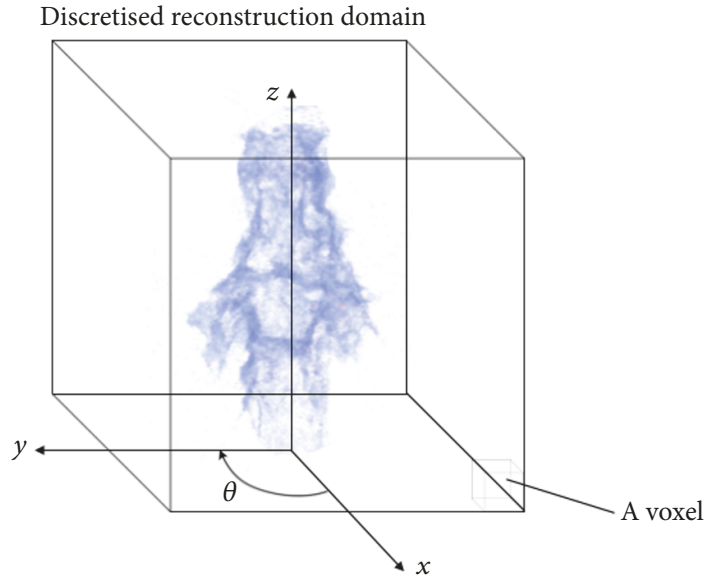

FIGURE 3: Reconstruction domain around the flame, discretized into voxels.

of $t_{\text {exp }}=0.1 \mathrm{~ms}$ was used. Images of the background signal (the scene without the flame) were obtained by each camera directly after the flame tests. Background correction was applied by subtracting the pixel intensities of the background images from the flame images.

\section{3D Instantaneous Reconstructions of the Flame}

All 24 views of the flame were used for the reconstructions. The flame images had a pixel resolution of $0.8 \mathrm{~mm}$ and contained 164 by 168 pixels in the horizontal and vertical directions respectively (providing a total of 27,552 projection measurements per view). The $3 \mathrm{D}$ reconstruction domain constituted 164 by 164 by 168 voxels in the $x y z$ directions, the coordinates are illustrated in Figure 3. Examples of the flame images obtained at different angles for one instantaneous time are shown in Figure 4. 

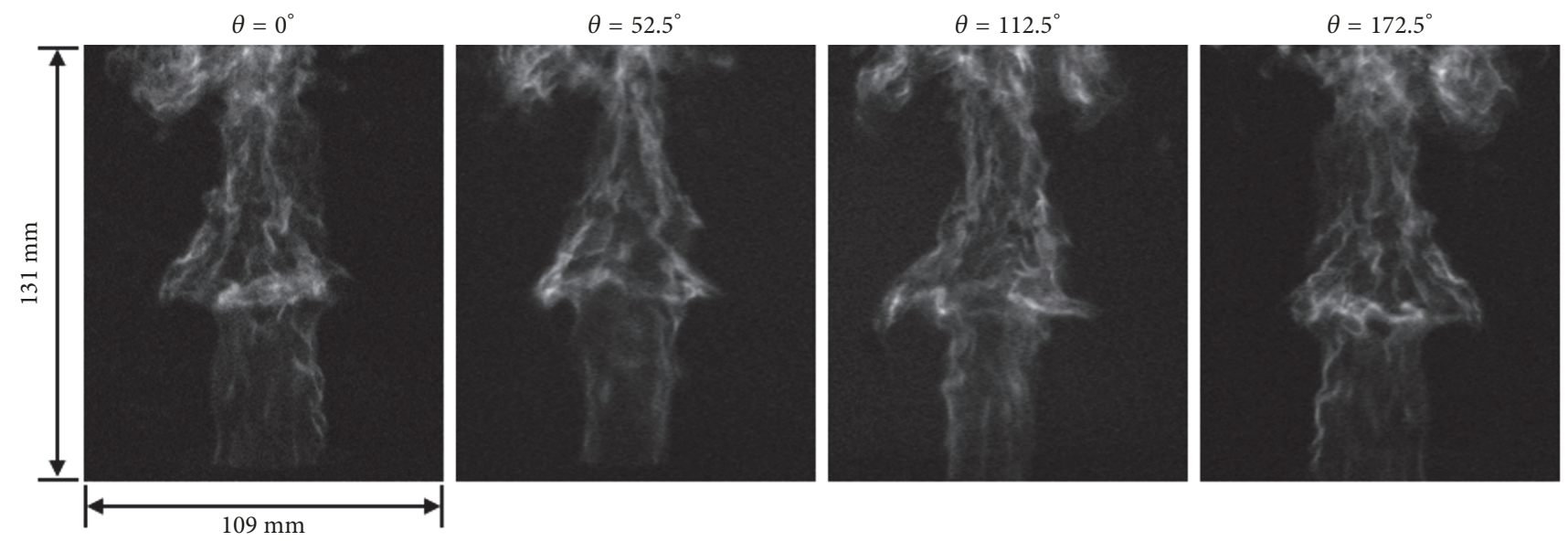

Figure 4: Examples of the flame projections from different angles, for the $83 \mathrm{~kW}$ flame.

$t_{1}$
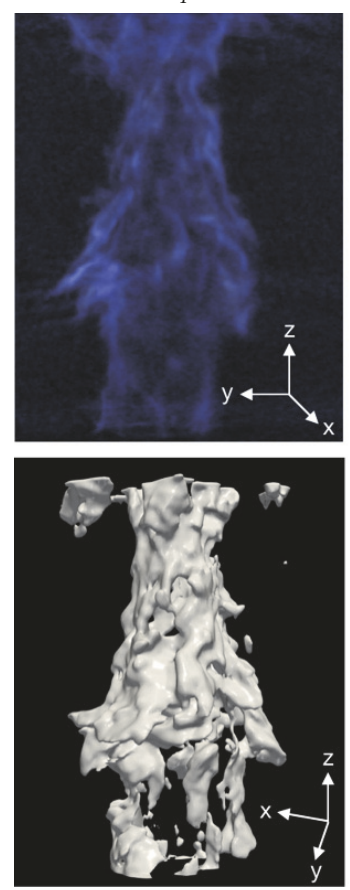

$t_{2}$
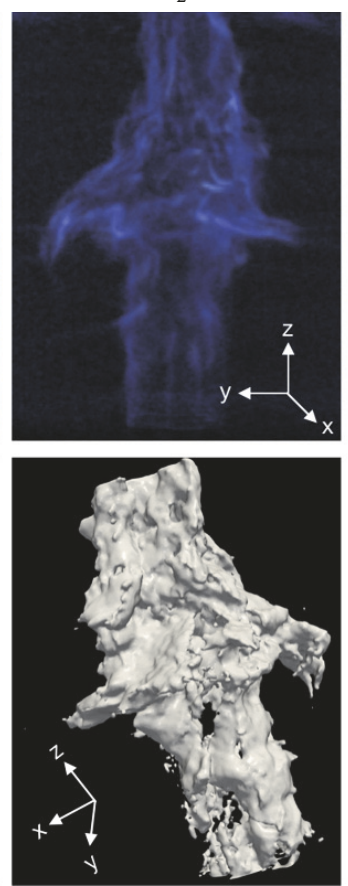

$t_{3}$
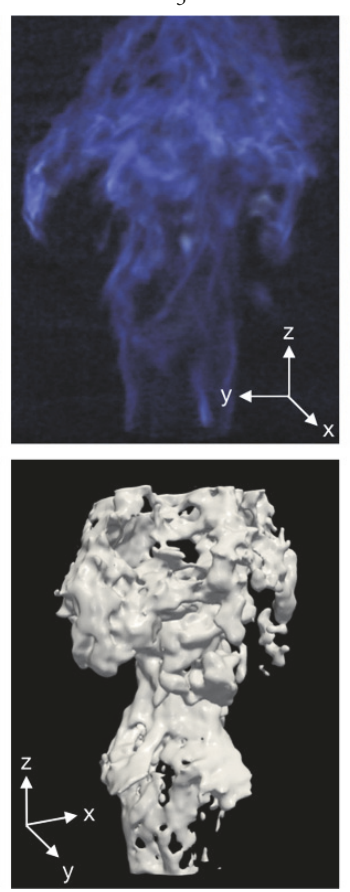

$t_{3}$, out-of-plane
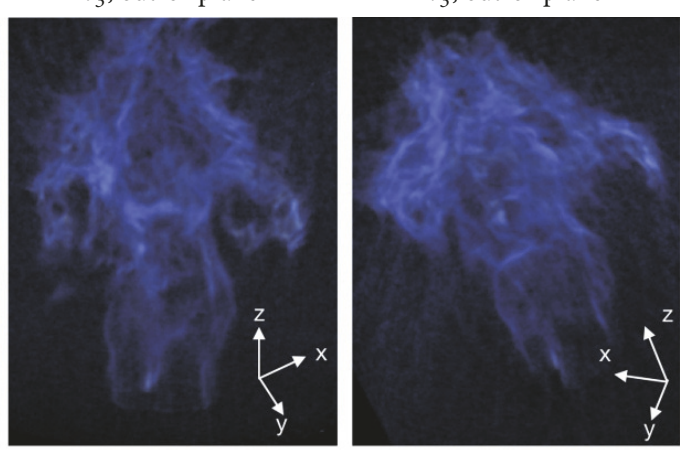

Figure 5: Volume-rendered views (top) and iso-surfaces (bottom) of the reconstructed $83 \mathrm{~kW}$ flame at different randomly chosen instances in time, shown from random angles that do not correspond to any view angle.

Volume-rendered views and iso-surfaces of the reconstructed $83 \mathrm{~kW}$ flame, for different instances in time, particularly chosen to reveal the shedding of large-scale structures in the dowstream direction, are shown in Figure 5. For one case, $t_{3}$, two further inclined views at two further random angles are illustrated. It is important to observe the field from angles that do not coincide with any of the original view angles, since these angles will have a bias towards better reconstruction quality.

Examples of the horizontal slices at different heights above the burner $z$, and vertical slices from the reconstructed fields are shown in Figures 6 and 7, respectively. Data is presented for both instantaneous and time-averaged flames (averages are calculated from 100 instantaneous snapshots). It is a particularly stringent test to check the horizontal slices from the reconstructed field since information was only provided to the algorithm from the vertical directions. The slices in Figure 6 demonstrate a good reconstruction quality that does not exhibit the artefacts which are typically seen in lowquality CTC results, such as parallel lines that cut through the domain. The instantaneous slices show that from very close to the burner exit the flame is highly wrinkled. The averaged flame reconstructions show the expected smooth shape but since the flames were very unsteady (as observed during the experiments), more than 100 instantaneous snapshots are presumably needed to produce a fully symmetric field. There 
$83 \mathrm{~kW}$
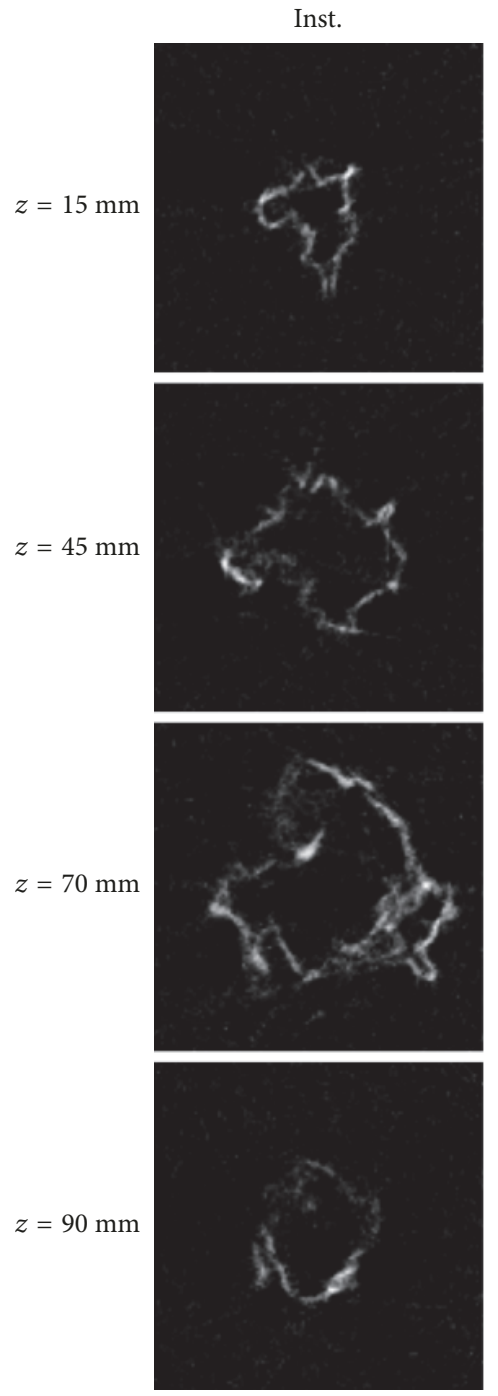
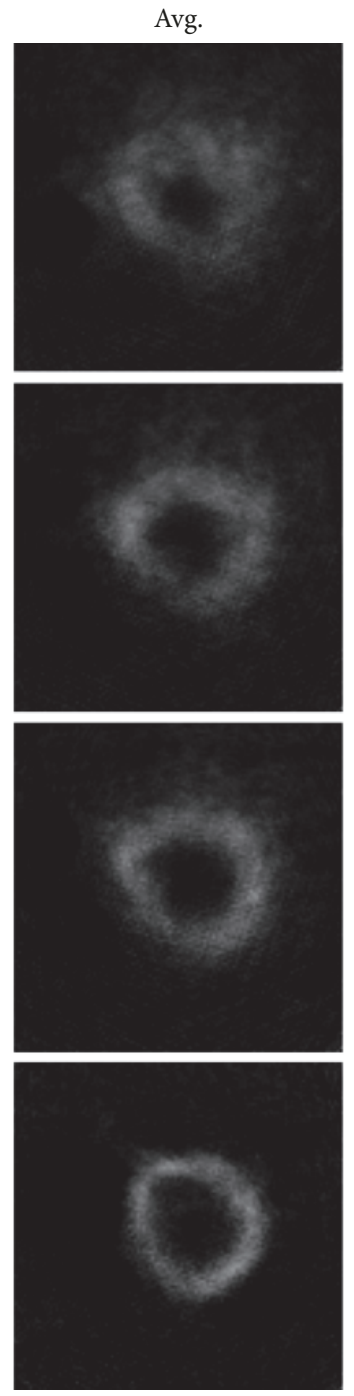

$105 \mathrm{~kW}$
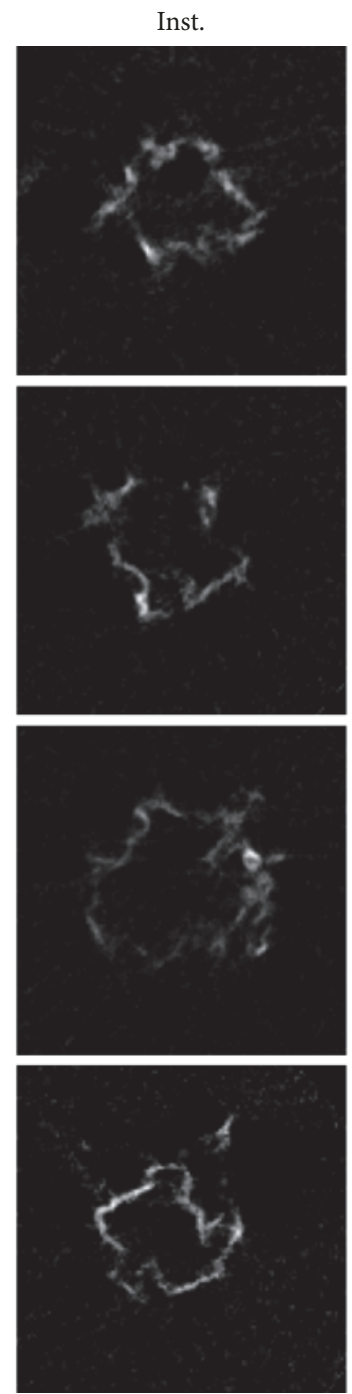
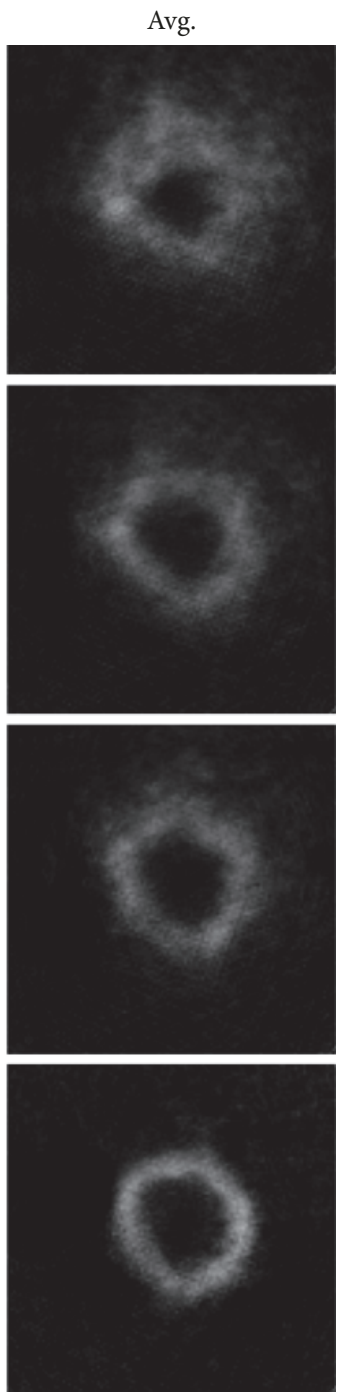

FIGURE 6: Horizontal slices from the reconstructed instantaneous and time-averaged (from 100 snapshots) flame images, at different heights above the burner $\mathrm{z}$.

does not appear to be a striking difference between the two different powered flames.

\section{Conclusions}

The chemiluminescence field of highly turbulent and unsteady industrial burner flames at the GWI were reconstructed for the first time using the full CTC technique that comprises an experimental setup with 24 low-cost CCD cameras (for capturing the flame chemiluminescence), and a tomographic algorithm that includes non-parallel perspective-corrected projections. The wrinkled flame shape in the instantaneous reconstructions and the smooth field in the time-averaged cases were revealed by looking at the volume-rendered views and different horizontal and vertical slices from the reconstructed fields. All the results showed a good reconstruction quality that can be achieved from the low-cost and versatile CTC technique, proving it to be a practical flame imaging method. Reconstructions from different instances in time showed that the flames shed large-scale structures in the downstream direction.

\section{Data Availability}

All the data, including raw flame images and reconstructed fields, that were used for the production of the manuscript is available within specially allocated hard drives in our department and can be accessed without restriction.

\section{Disclosure}

This work was presented at the 3rd General Meeting and Workshop on SECs in Industry COST Action 1404 of the 
$83 \mathrm{~kW}$
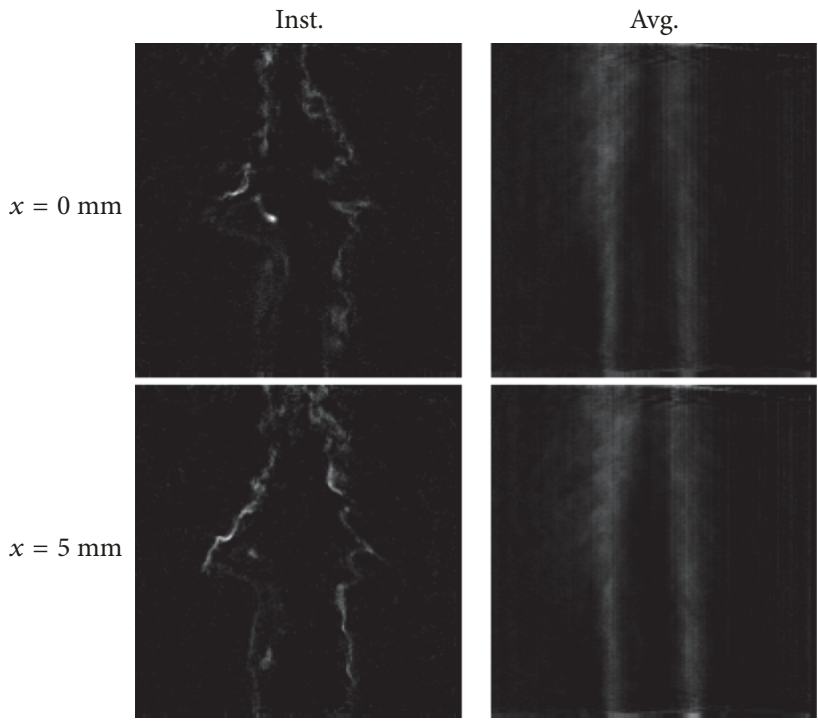

$105 \mathrm{~kW}$
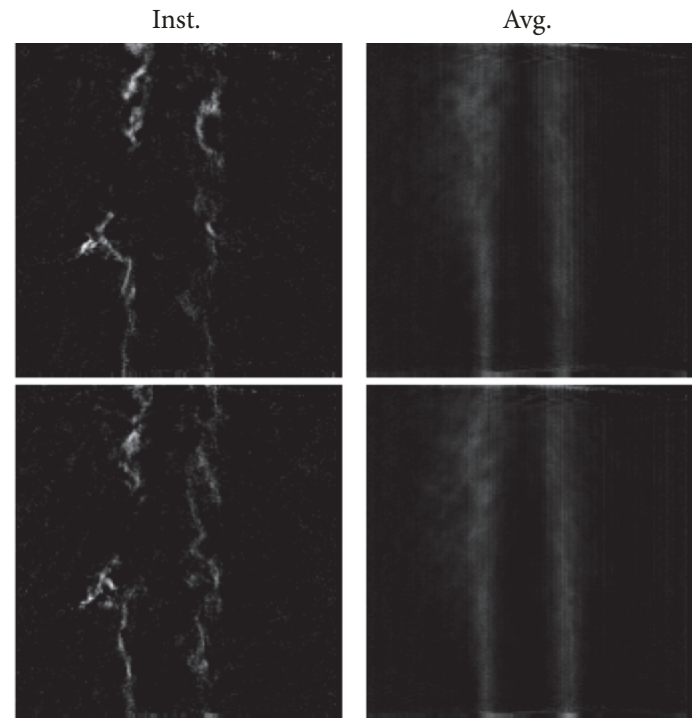

FIGURE 7: Vertical slices from the reconstructed instantaneous and time-averaged (from 100 snapshots) flame images, at the burner centreline and one neighbouring plane.

European Cooperation in Science and Technology, Prague (Oct. 2017).

\section{Conflicts of Interest}

The authors declare that they have no conflicts of interest.

\section{Acknowledgments}

The authors are grateful for the funding from the Ministerium für Innovation, Wissenschaft und Forschung des Landes Nordrhein-Westfalen, and the support by the Open Access Publication Fund of the University of Duisburg-Essen.

\section{References}

[1] B. Peterson, E. Baum, B. Böhm, V. Sick, and A. Dreizler, "Highspeed PIV and LIF imaging of temperature stratification in an internal combustion engine," Proceedings of the Combustion Institute, vol. 34, no. 2, pp. 3653-3660, 2013.

[2] K. Y. Cho, A. Satija, T. L. Pourpoint, S. F. Son, and R. P. Lucht, "High-repetition-rate three-dimensional $\mathrm{OH}$ imaging using scanned planar laser-induced fluorescence system for multiphase combustion," Applied Optics, vol. 53, no. 3, p. 316, 2014.

[3] J. Floyd, P. Geipel, and A. M. Kempf, "Computed Tomography of Chemiluminescence (CTC): instantaneous 3D measurements and Phantom studies of a turbulent opposed jet flame," Combustion and Flame, vol. 158, no. 2, pp. 376-391, 2011.

[4] K. Mohri, S. Göers, J. Schöler et al., "Instantaneous 3D imaging of highly turbulent flames using computed tomography of chemiluminescence," Applied Optics, vol. 56, no. 26, pp. 73857395, 2017.

[5] M. M. Hossain, G. Lu, D. Sun, and Y. Yan, “Three-dimensional reconstruction of flame temperature and emissivity distribution using optical tomographic and two-colour pyrometric techniques," Measurement Science and Technology, vol. 24, no. 7, Article ID 074010, 2013.

[6] Y. Yan, T. Qiu, G. Lu, M. M. Hossain, G. Gilabert, and S. Liu, "Recent advances in flame tomography," Chinese Journal of Chemical Engineering, vol. 20, no. 2, pp. 389-399, 2012.

[7] Floyd, J., Computed tomography of chemiluminescence: a 3D time resolved sensor for turbulent combustion. 2009, Imperial College London.

[8] J. Floyd and A. M. Kempf, "Computed Tomography of Chemiluminescence (CTC): high resolution and instantaneous 3-D measurements of a matrix burner," Proceedings of the Combustion Institute, vol. 33, no. 1, pp. 751-758, 2011.

[9] K. Mohri and A. Kempf, "Computed tomography of chemiluminescence for 3D reconstructions of quasi-steady premixed flames," in in Combustion Institute (British section) meeting, talk and poster, London, Imperial College, 2014.

[10] K. Mohri and A. Kempf, "Computed tomography of chemiluminescence in asymmetric unsteady premixed flames," in 27th Deutscher Flammentag, VDI-Berichte Nr. 2267, ClausthalZellerfeld, 2015.

[11] J. R. Howell, R. Siegel, and M. P. Mengüc, Thermal radiation heat transfer, CRC Press, Taylor \& Francis Group, Boca Raton, Florida, USA, 5 edition, 2010.

[12] K. J. Daun, S. J. Grauer, and P. J. Hadwin, "Chemical species tomography of turbulent flows: Discrete ill-posed and rank deficient problems and the use of prior information," Journal of Quantitative Spectroscopy \& Radiative Transfer, vol. 172, pp. 58-74, 2016.

[13] R. Gordon, "A tutorial on art (algebraic reconstruction techniques)," IEEE Transactions on Nuclear Science, vol. 21, no. 3, pp. 78-93, 1974. 


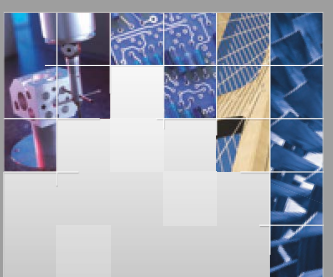

\section{Enfincering}
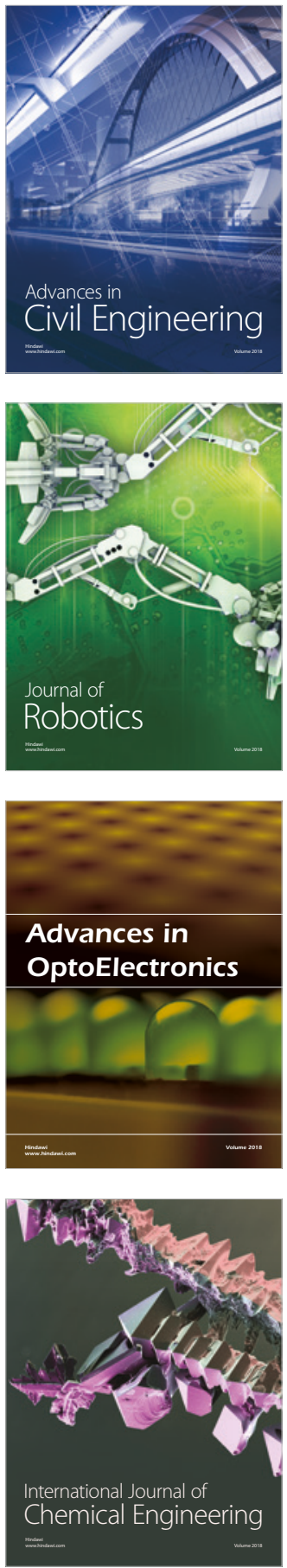

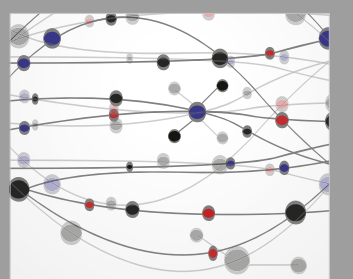

\section{Rotating \\ Machinery}

The Scientific World Journal

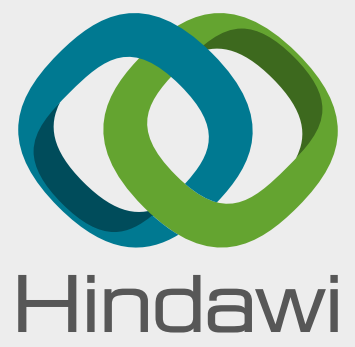

Submit your manuscripts at

www.hindawi.com
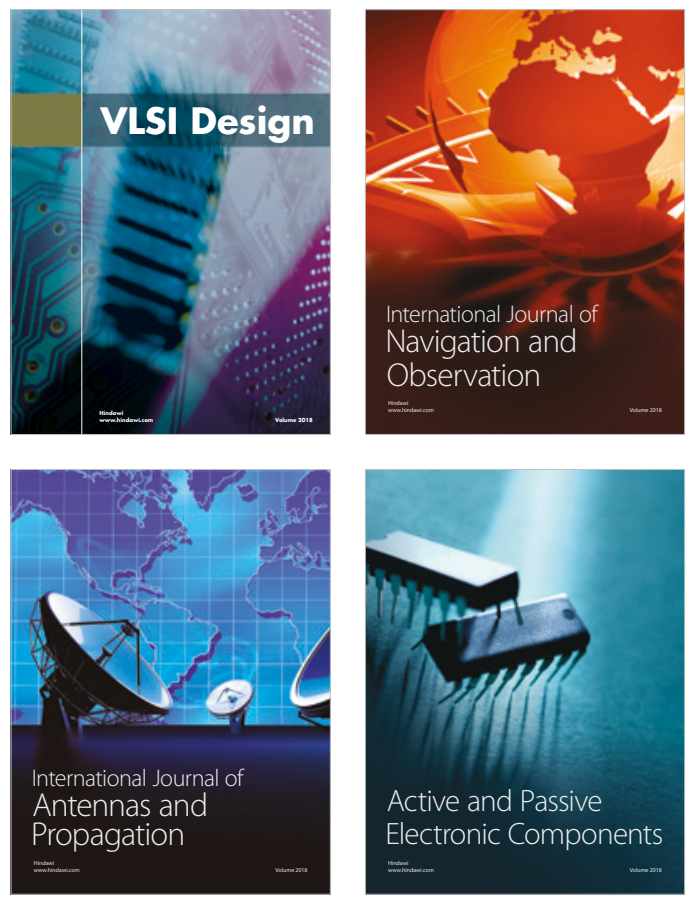
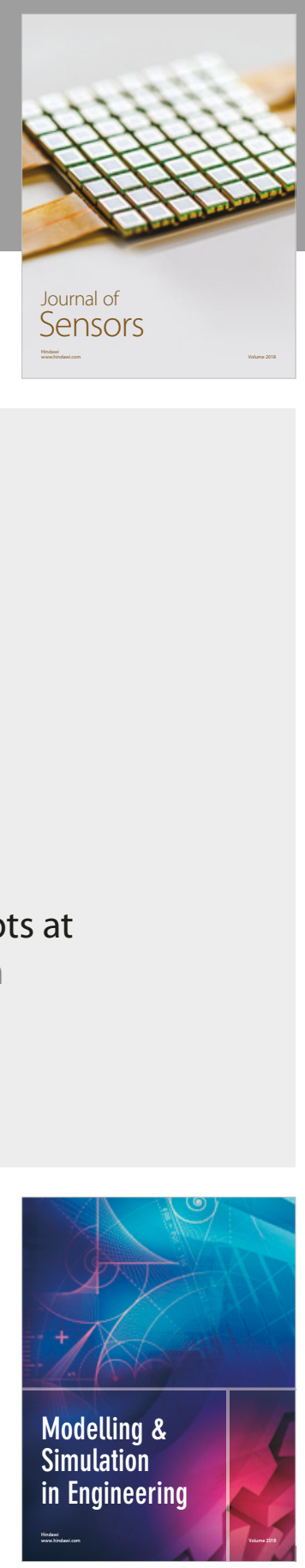

\section{Advances \\ Multimedia}
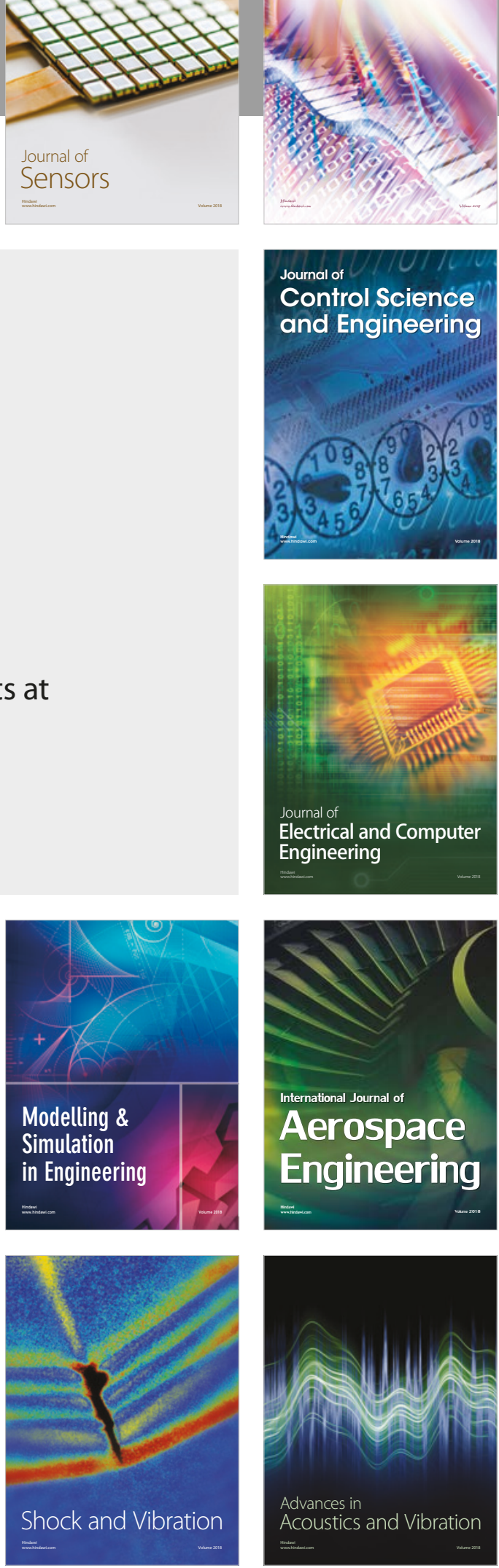